\title{
Star Formation in Expanding Shells: When and Where
}

\author{
S. Ehlerová ${ }^{1}$, J. Palouš ${ }^{1}$, Ch. Theis ${ }^{2}$, and G. Hensler ${ }^{2}$ \\ 1 Astronomical Institute, Academy of Sciences of the Czech Republic, Bočni II \\ 1401, 14139 Prague, Czech Rep. \\ ${ }^{2}$ Institut für Astronomie und Astrophysik, Univ. Kiel, Germany
}

\begin{abstract}
The fragmentation of expanding shells and subsequent star formation are analyzed using an analytical model and computer simulations. We discuss the role of the sound speed in the ambient interstellar medium and the influence of the finite thickness of the gaseous disk.
\end{abstract}

\section{Introduction}

Fragmentation of dense gaseous shells expanding around OB associations and subsequent formation of molecular clouds is one of the ways how the star formation propagates in galaxies. The amount of the energy input from stellar winds and supernovae in an OB association, density distribution of the interstellar medium, random motions in the ambient medium, galactic rotation and $K_{z}$ force are main factors influencing the evolution of shells. The relation of these properties to the propagating star formation is discussed in the present contribution.

\section{Fragmentation in the Thin Shell Approximation}

Initially, a multi-supernova remnant follows a quasi-adiabatic stage. Once radiative losses become important a thin and cold shell forms. It expands sweeping the ambient medium until it slows down to a velocity comparable to the random motions in the interstellar medium. The latter phase of expansion can be described by an infinitesimally thin shell approximation (Ostriker \& McKee, 1988; Bisnovatyi-Kogan \& Silich, 1995).

An analytical self-similar solution is in this paper complemented by a numerical computer code (Ehlerov et al, 1997). It gives us an opportunity to study effects of the stratified distribution of the interstellar medium, galactic rotation, $K_{z}$ force and cooling from inside the hot bubble.

Using the linear perturbation theory, Elmegreen (1994) described fragmentation in an expanding thin shell. A density fluctuation in the shell can grow if two conditions are fulfilled: 
1) growth rate condition given in Equation (2) by Theis et al. in these proceedings, and

2) dimension condition $\eta=\pi G \Sigma R / c>2 \pi$,

where $R$ is the diameter of the shell, $\Sigma$ is the shell column density, $c$ is the sound speed inside the shell, and $G$ is the constant of gravity.

The time, when the two conditions are fulfilled for the first time, is called the instability time $t_{b}$. For times $t>t_{b}$ we define the fragmentation integral $I_{f}$ :

$$
I_{f}(t)=\int_{t_{b}}^{t} \omega\left(t^{\prime}\right) d t^{\prime}
$$

where $\omega$ is the maximum growth rate of a transverse perturbation in the shell. At the time $t_{f}$, when the fragmentation integral $I_{f}\left(t_{f}\right)=1.0$, fragments are in a non-linear phase of the evolution and the linear approximation of growth is no longer valid. At this time fragments are well developed, subsequently forming new clouds.

To form molecules fragments must be self-shielded against the ionizing radiation. It happens when

3) The self-shielding condition $N \geq N_{\text {crit }}=5 \times 10^{20} Z_{\odot} / Z \mathrm{~cm}^{-2}$ is fulfilled.

(Franco \& Cox, 1986), where $N$ is the particle column density of the shell, $Z$ is the metallicity.

In a static, homogeneous medium with a negligible external pressure fragmentation conditions 1) and 2) may be rewritten to an analytical self-similar solution as functions depending on the energy input rate from the $\mathrm{OB}$ association, density and sound speed of the ambient medium (Theis et al., these proceedings).

However, the analytical solution is restricted to spherical or cylindrical symmetries. The spherical case is probably a good approximation for shells inside GMCs or shells in galaxies with thick gaseous disks. In spiral galaxies with thin gaseous disks the cylindrical symmetry may apply but there the galactic differential rotation plays a role. Also, some time after the formation of an $\mathrm{OB}$ association, which may be shorter than time $t_{b}$, all massive stars have exploded as supernovae and the energy input is stopped. Another problem, which is not taken into account in the analytical solution, concerns the shell expansion velocity $v$. When $v$ decelerates to the sound speed in the ambient medium $c_{a m b}$, the shell begins to propagate as a sound wave. The blast wave approximation is no longer valid. The way how to take mentioned effects into account is to use numerical computer simulations (Ehlerováet al, 1997).

\section{Sound Speed and Stratified Medium}

We have already indicated the importance of the sound speed in the ambient medium. If the shell reaches the subsonic expansion velocity before the time 


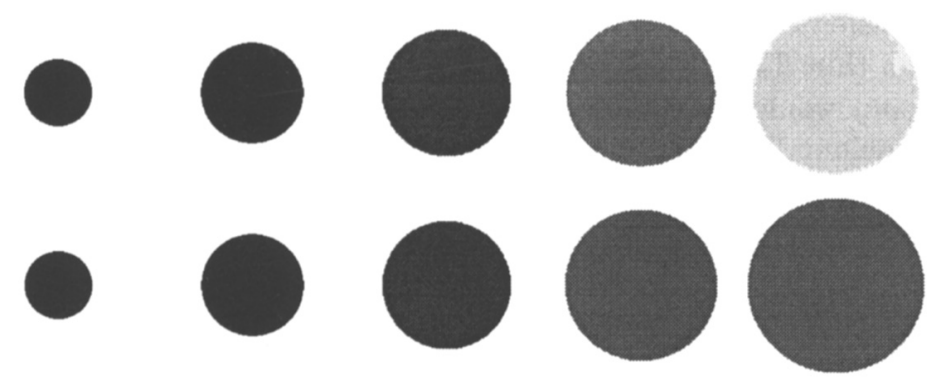

Fig. 1. Numerical simulations: shells in a homogeneous medium with a density of $1 \mathrm{~cm}^{-3}$ and a sound speed of $5 \mathrm{~km} \mathrm{~s}^{-1}$ (upper panel) and $10 \mathrm{~km} \mathrm{~s}^{-1}$ (lower panel) at times 10, 20, 30, 40 and 50 Myr. Black color indicates stability, grey instability.

$t_{b}$, it never fragments. Even if the fragmentation starts, the fragments may reexpand and the shell remains stable. Illustrative case is given in Fig. 1, which shows two shells with the same energy input into the ambient medium with the density of $1 \mathrm{~cm}^{-3}$. The two cases differ in the sound speed in the ambient medium, which is $5 \mathrm{~km} \mathrm{~s}^{-1}$ or $10 \mathrm{~km} \mathrm{~s}^{-1}$. In the first case the shell completely fragments while in the second case it reexpands and remains stable.
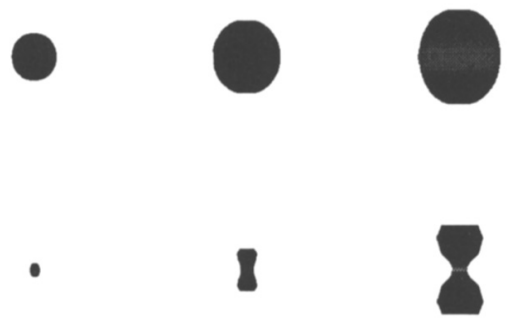
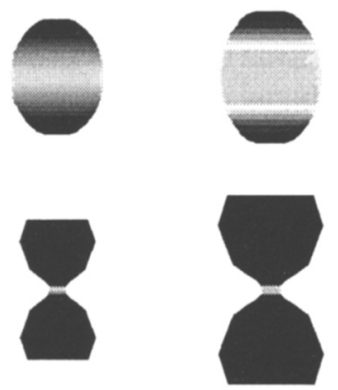

Fig. 2. Numerical simulations: shells expanding in a thick disk (upper panel) and thin disk (lower panel) at times 10,20,30,40 and $50 \mathrm{Myr}$. Black color indicates stable parts, grey color unstable ones.

Shells in galaxies evolve in a gaseous disk of a certain thickness. Different thicknesses lead to different fragmentation properties, which is demonstrated in Fig. 2 showing the evolution of two shells: one in a thick gaussian disk (the scale height of $500 \mathrm{pc}$ ), the other in a thin gaussian disk (the scale height of $200 \mathrm{pc}$ ). At the time when $I_{f}$ in the most unstable parts reaches the value 1.0 , nearly the whole surface of the shell in the thick disk is unstable, while in the thin disk the shell develops only a tiny instability ring. In absolute values, 
a z-dimension of unstable parts in a thick disk case is about 4 times larger than in a thin disk case. A shell in the disk with an even lower scale height $(\sim 100 \mathrm{pc})$ would be stable during the whole evolution. An explanation of this behaviour lies in a blow-out effect. Once the upper parts of the shell break through the disk, a great deal of the internal energy escapes to the halo leading to a rapid decrease of the internal pressure and, consequently, to a rapid deceleration of parts of the shell near the galactic plane.

\section{Conclusions and Summary}

The sound speed in the ambient medium is one important quantity influencing the fragmentation. A critical value dividing the fragmentating and non-fragmentating shells lies between $(5-10) \mathrm{km} \mathrm{s}^{-1}$, which is in the range of random velocities measured in galaxies. Another important quantity is the thickness of the HI disk: in thin disks, shells are gravitationally stable or develop only a narrow instability belt near the galactic equator; in thick disks, a great part of the shell is unstable and new clouds may form also off the galactic plane. This corresponds to conclusions presented by Theis et al. in these proceedings based on analytical self-similar solution: in a thick gaussian disk the shell fragments in shallow parts of the density profile, where is the density gradient flatter than an isothermal profile, whereas in a thin gaussian disks the density gradient increases to values larger than an isothermal profile before the gravitational fragmentation happens. We can conclude that in dwarf galaxies similar to LMC, SMC or HoII, the fragmentation in expanding shells occurs nearly everywhere. Therefore, the star formation can easily propagate. In spiral galaxies similar to Milky Way, the fragmentation of shells is restricted to a narrow strip at the galactic plane. Thus the star formation can propagate only to a limited portion of the galaxy.

Acknowledgements. The authors gratefully acknowledge financial support by the Grant Agency of the Czech Republic under grant no. 205/97/0699 and by the Deutsche Forschungsgemeinschaft under grant no. 436 TSE 17/5/96.

\section{References}

Bisnovatyi-Kogan G.S. \& Silich S.A., 1995, Rev. Mod. Phys. 67, 661

Ehlerová S., Palouš J. Theis Ch. \& Hensler G., 1997, submitted to A\&A

Elmegreen B.G., 1994, ApJ 427, 384

Franco J. \& Cox D.P., 1986, ApJ 273, 243

Ostriker J.P. \& McKee C.F., 1988, Rev. Mod. Phys. 60, 1

Theis Ch., Ehlerová S. \& Palouš J., 1997, this volume 